\title{
Chance for snowballs in hell
}

David A. Paige

IN October 1991, when radar astronomers ${ }^{1.2}$ first announced that the anomalous reflections they observed at the poles of the planet Mercury were due to the presence of water ice deposits, the news was greeted with a fair amount of scepticism ${ }^{3}$. However, new higherresolution radar maps reported on page 213 of this issue ${ }^{4}$ leave little room for doubt: they show that the regions of anomalous reflectivity are localized within small areas whose positions can be made to coincide exactly with the positions of high-latitude impact craters. According to theory, the permanently shadowed floors of these craters are the only places on Mercury that might be cold enough to trap water ice for billions of years 5.6 .

How can water ice be stable on the closest planet to the Sun? Surfaces on Mercury that are exposed to the Sun's direct rays become very hot, reaching temperatures as high as $700 \mathrm{~K}$ at the equator when the planet is closest to the Sun. However, because Mercury's spin axis is almost exactly perpendicular to its orbital plane, the situation at Mercury's poles is very different. To an observer at one of the poles, the Sun never rises or sets, but remains perched at the horizon throughout the year.

Thermal models show that if the surface of Mercury had no topographic relief, then the glancing solar rays that reach the poles would still be strong enough to result in maximum temperatures of about $170 \mathrm{~K}$ - cold by terrestrial standards, but still not cold enough to prevent the rapid evaporation of any water ice deposit 6 . However, images obtained by the Mariner 10 spacecraft as it flew by the planet in 1974 and 1975 show that the surface is far from flat; it is pockmarked with impact craters. At high latitudes, crater rims provide shade for the walls and floors within, creating regions that never receive any direct sunlight. The temperatures of these permanently shadowed regions are determined primarily by the fluxes of scattered solar radiation and emitted infrared radiation from the crater walls. Within larger craters, the annual maximum surface temperatures within these permanently shadowed regions should routinely be as low as $100 \mathrm{~K}$ (refs 5,6 ). At these temperatures, the vapour pressure of water ice is so low that once established, ice deposits should be stable for billions of years - just as they are on the surfaces of icy satellites in the outer Solar System.

Two pieces of good fortune have allowed the Jet Propulsion Laboratory's Goldstone antenna with the Very Large Array $^{1}$, and the Arecibo facility in Puerto $\mathrm{Rico}^{2}$ where the new results were also obtained $^{4}$, to reveal the presence of ice in these dark, hidden regions. The plane of Mercury's orbit about the Sun is inclined by seven degrees relative to that of the Earth, which makes it possible for Earthbased radars to 'see' into the interior regions of these craters despite the fact that they are never directly illuminated. Furthermore ice deposits on Mercury give distinctive, bright, depolarized radar

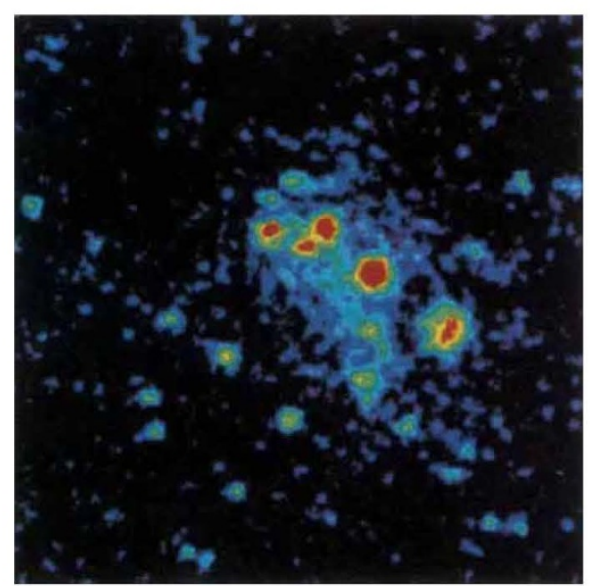

Radar image of the north polar region of Mercury. Many of the bright spots have been identified with known impact craters. The strong echoes may be backscatter from ice deposits in the crater floors.

echoes which make them stand out in stark contrast to their surroundings. Similar echoes have been observed for ice deposits on Mars and the icy satellites of Jupiter?

Theories to explain the depolarized echoes work only if the ice contains few absorbing contaminants such as rocks and soil, and if it is several times thicker than the radar wavelength ${ }^{8}$. The observations do not rule out the possibility that the ice is covered by a thin layer of soil, which would be largely transparent at radar wavelengths. Aside from these few constraints, little else is known about the physical properties of these deposits.

What can be said about the composition, origin and ages of the material that is cold-trapped at Mercury's poles? The fact that the geographical distribution of the observed radar features agrees so closely with that expected for cold-trapped water ice deposits, combined with water's high cosmic abundance, leaves little doubt that water is one of the main volatile constituents. In fact, from what is known at present, one could easily argue that all available niches for stable water ice on Mercury are full. However, there is a possibility that other, more volatile species are stable within the coldest permanently shadowed regions. From past considerations of sources for possible ice deposits at the poles of the Moon ${ }^{9}$, the most likely sources for the volatile materials now trapped at Mercury's poles include degassing from the planet's interior, comet and meteorite impacts, and the production of water by the reduction of iron in surface soils by the implantation of hydrogen in the solar wind ${ }^{8}$. As the timescales for the supply and loss of volatile materials from these cold regions are difficult to constrain, it is not yet possible to determine whether Mercury's ice deposits are ancient remnants of the planet's early history, or dynamic, contemporaneous features that are actively being replenished.

The presence of ice at Mercury's poles is an exciting new discovery that will have an impact on future efforts to understand and explore the Solar System. Both NASA and the European Space Agency are currently considering low-cost Mercury flyby and Mercury orbiter missions designed to fill gaps in our present knowledge, including the fact that half the planet's surface has never been imaged by spacecraft. The independent detection of ice, and a better understanding of the processes responsible for its presence, should receive high priority in the planning and design of these Mercury missions.

The new results should also spur efforts to search for ice at the poles of the Moon. Watson, Murray and Brown ${ }^{10}$ proposed in 1961 that ice might exist in permanently shadowed craters near the lunar poles, but so far no lunar spacecraft, not even the Clementine orbiter launched earlier this year (and now malfunctioning), has been well equipped to detect it. If ice deposits were found on the Moon, they would be exciting targets for robotic exploration, as well as resources that could improve the chances of long-term human habitation. For present-day space scientists and space explorers, though, the radar results demonstrate something equally valuable. The Solar System still contains many secrets that lie waiting to be discovered some hidden in places where the Sun never shines.

David A. Paige is in the Department of Earth and Space Sciences, University of California, Los Angeles, 3806 Geology Building, Los Angeles, California 90024, USA.

1. Slade, M. A., Butler, B. J. \& Muhleman, D. O. Science 258, 635-640 (1992)

2. Harmon, J. K. \& Slade, M. A. Science 258, 640-643 (1992)

3. Chapman, C. R. Nature 354, 504-505 (1991)

4. Harmon, K etal Nature 369, 213-215 (1994)

5. Paige, D. A., Wood, S. E. \&Vasavada, A. R. Science 258. 643-646(1992)

6. ingersoll, A. P., Svitek, T. \& Murray, B. C. Icarus 100 $40-47(1992)$

7. Muhleman, D. O., Butler, B. J., Grossman, A. W. \& Siade, M. A. Science 253, 1508-1513(1991)

8. Butler, B. J., Muhleman, D. O. \& Slade, M. A. J. geophys. Res. 98, 15003-15023 (1993).

9. Arnold, J.R. J. geophys. Res. 84, 5659-5668 (1979).

10. Watson, K., Murray, B. C. \& Brown, H. J. geophys. Res. 66. 3033-3045 (1961). 